\title{
Pneumatic Muscle-Actuated Adjustable Compliant Gripper System for Assembly Operations
}

\author{
Tudor Deaconescu* - Andrea Deaconescu \\ Transilvania University of Brasov, Romania
}

The aim of this paper is to present and discuss an innovative, constructive solution for a gripper system that can be attached to an industrial robot for assembly operations. The construction of this gripper system is based on a linear pneumatic muscle used as the actuator and the transmission of motion by gear-and-rack mechanisms. Air compressibility renders pneumatic muscle behaviour inherently compliant; this favours automated assembly applications as it allows the correction of inevitable lateral and angular misalignments in mating operations. Therefore, the jamming of a peg-like object can be avoided when this is introduced into a hole with tight clearance. In addition to the construction of the gripper system, this paper discusses its actuation, as well other characteristics.

Keywords: assembly, compliance, grippers, manipulators, pneumatic muscles, stiffness

Highlights

- An adjustable compliant gripper system for assembling robots is proposed.

- $\quad$ The utilised motor, namely the pneumatic muscle, benefits from adjustable compliance and can adapt its operational behaviour between two limits, ranging from rigid (for high accuracy positioning) to compliant.

- $\quad$ The structural diagram of the gripper system, its construction and its pneumatic control diagram are presented.

- The variation of the gripper system stiffness and compliance are both demonstrated.

\section{INTRODUCTION}

Grippers are defined as those components of robots that facilitate temporary contact with a manipulated object, ensuring its position and orientation during transport, assembly or while conducting certain specific tasks. At present, grippers tend to become increasingly sophisticated, more complex, so as to meet varied requirements. Complex construction, however, entails high costs, often unacceptable in a competitive industrial scenario [1]. For this reason, current industrial applications typically deploy simple and reliable gripper systems, while for research purposes complex mechanical hands are proposed.

One modality of classifying gripper systems is by their destination. According to this criterion, while gripper systems can be designed for industrial applications, another category includes mechanical hands used mostly for research. In principal, industrial gripper systems are described by the developed force and their structural rigidity. These characteristics can be ensured by conceiving grippers with few degrees of freedom, thus facilitating system reliability and low costs. Mechanical hands resemble natural systems and are thus characterized by better compliance and dexterity, which makes them eligible for dexterous applications [2].

Conceiving a novel gripper system entails correctly defining its required functional characteristics. Requirements, such as the developed force, stiffness/compliance, dexterity, and the number of degrees of freedom, depend on the application for which the gripper is designed. Studies have revealed that if the object gripping ability of a five-finger mechanical hand is of $100 \%$, then a four-finger mechanical hand will have $99 \%$ of its ability, a threefinger one about $90 \%$, while this ability will be of only $40 \%$ in a hand with two "fingers" [3].

In applications in which emphasis is laid on safe seizing, the risk of damaging the seized object can be significantly reduced by drastically increasing sensorization and by adequate design of the control diagrams. An alternative to over-sensorization is using variable stiffness actuators (VSAs), also known as adjustable compliant actuators (ACAs). These actuators are used because of their benefits, including the capacity of minimizing the effects of mechanical shocks, the safety of interaction with human operators or the ability to store and release energy into passive elastic elements [4].

The utilization of adjustable compliant actuators ensures the adaptability of the gripper system to concrete work situations that may differ from the initially envisaged ones. A mechanical system is defined as adaptive when it has the capacity to adequate responding to new situations within the limitations given by the mechanical band width determined by the system's elasticity and inertia, as well as by the internal friction. In such a system, the presence of sensors or complex controllers is not 
necessary, as it is the mechanical system itself that provides the required adaptive behaviour [5].

In the case of gripper systems, adaptive behaviour entails adaptation to either the (irregular) shape of the seized object, or to the constraints created by the environment it evolves within (for example positioning the gripper relative to the object). In recent years, numerous efforts have been made to create new gripper systems with easily achievable and controllable constructions, adaptable to environmental constraints. Thus, paper [6] presents a highly compliant robotic hand actuated via a novel pneumatic actuator, called PneuFlex. Paper [7] discusses an object-grasping strategy with directional position uncertainty, and [8] presents a tendon-driven robotic gripper performing fingertip and enveloping grasps. In [9], the authors describe a novel adaptive and compliant grasper that can grasp objects spanning a wide range of size, shape, mass, and position/ orientation using only a single actuator.

Robot applications for assembly entail the utilization of adjustable compliant gripper systems. This has become desirable because, while at present, very good positioning precisions can be obtained, assembly errors caused by machine inaccuracy (tolerances), vibration, effects of contamination, etc., will still occur. The deployment of a robot arm of lesser positioning precision and combined with a compliant gripper system provides a more costeffective solution.

In manual assembly, the above difficulties can be easily overcome by the human operator drawing upon the brain's reasoning capacity, expertise, experience and the dexterity of their fingers (the fingers' compliance). All these are completed by closed loop control provided by the link between the senses and the brain [10].

In automated assembly, the above disadvantages can be prevented by either uncontrolled (passive) mating mechanisms: remote centre compliance mechanisms (RCC) or controlled (active and passive) mating mechanisms, and instrumented remote centre compliance (IRCC) mechanisms [10].

An RCC mechanism is a passive mechanical device that facilitates automated assembly by correcting inherent lateral or angular misalignments in mating operations. Thus, the jamming of a peg-like object can be avoided when this is introduced into a hole with tight clearance.

The construction of gripper systems for automated assembly includes an RCC device that ensures the adaptive behaviour required for compensating the misalignments of mating parts.
The need for developing gripping systems that allow adaptive behaviour has yielded numerous solutions of actuators, sensors and structural design. Nature is an endless source of inspiration for engineers and holds many examples of gripper systems that can become starting points for practical applications. A few such examples of natural grippers are bird claws and beaks, elephant trunks, octopus tentacles, each using mechanical contact forces. While in natural systems these forces are generated by muscles, artificial grippers use motors to develop forces [2].

The above examples suggest that innovative, bio-inspired gripper systems should be made from light components that can be flexibly structured, that ensure a large useful load-to-eigenweight ratio, that have integrated position and force control, all at an affordable price. It is in this sense that this paper presents and discusses the construction and performance of a novel gripper system actuated by a pneumatic muscle type motor, a bioinspired system similar to human and animal muscles.

The second part of the paper presents the actuator used in the construction of the gripper system for automated assembly, with an emphasis on a characteristic of the pneumatic muscle, namely compliance. The third part of the paper describes the construction of the gripper system and its actuation, and the fourth part presents the experimental results obtained for system compliance. The paper continues with the presentation and discussion of a case study of a mating operation (peg into hole) and is finalized by a section for conclusions.

\section{THE PNEUMATIC MUSCLE - A COMPLIANT ACTUATOR}

The pneumatic muscle mimics the behaviour of natural muscle fibres, and is characterized by low weight, reduced overall dimensions, a small massto-power unit ratio, the capacity to absorb shocks, as well as compliance. When fed compressed air, the pneumatic muscle contracts axially and deforms radially. The compressibility of air makes it inherently compliant (passive compliant actuator), and it behaves in a spring-like fashion, that can store energy.

The emergence of pneumatic muscles has led in certain applications to the replacement of single-acting pneumatic cylinders. Compared to these, pneumatic muscles of the same size have an eight-times lower weight, while generating a ten-times greater force. As in pneumatic muscles, stick-slip does not occur, motions of small amplitude and reduced velocity are achievable, thus proving their superior dynamic behaviour compared to pneumatic cylinders. 
Using pneumatic muscles in grippers ensures obtaining of light and flexible constructions capable of safely manipulating objects. Firm gripping of an object without damaging it is ensured by a special characteristic of pneumatic muscles, known as compliance.

Compliance, the inverse of rigidity is an actuator's characteristic of allowing deviations from a certain position of equilibrium when the system is subject to the action of exterior forces. Actuators are adjustable compliant when their stiffness is not constant, which is the case when the dependency between the developed force and the displacement is non-linear. Pneumatic muscles (bio-inspired actuation systems) fit this description, due to their adaptive compliant behaviour, materialized by the possibility of continuous variation of the stiffness [11].

The utilization of pneumatic muscles in the construction of gripper systems remains in an incipient stage, because of the relatively recent emergence of these actuators. An example of a pneumatic muscleactuated gripper system is the device called Power Gripper manufactured by Festo (Germany), based on the way birds seize objects with their beaks [12]. The construction of the gripper is based on Watt linkages, and due to the deployment of a light motor (a pneumatic muscle) the system has a well-developed force-to-eigenweight ratio.

Other such achievements can be found in literature [13] and [14].

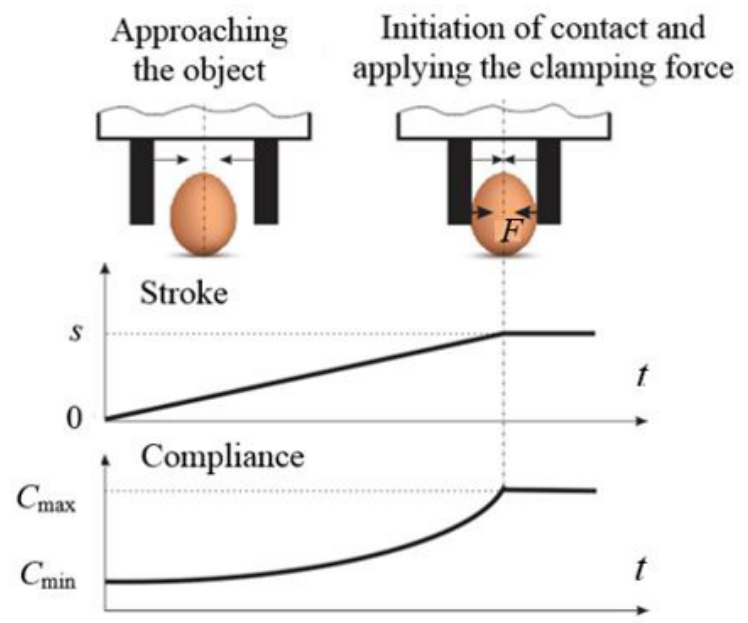

Fig. 1. Variation versus time of jaw stroke and system compliance

Fig. 1 presents certain standard phases of the gripping process, namely approaching the targeted object, initiation of contact and securing the seized object between the jaws by increasing the clamping force. For these phases graphs that describe the evolution versus time of the jaw stroke and of the compliance are presented. It can be noticed that once contact has been made with the targeted object, in order to avoid deformation of the object, the jaws remain motionless in position; thus, in this position of the jaws a maximum compliance has to be reached, such as to allow the correction of misalignments.

The pneumatic muscle is a passive compliant actuator, whose compliance is dependent on the stroke. For this reason, the compliance varies (increases) inevitably over the entire carried-out stroke, even before contact occurs between the jaw and object to be seized. Significant is the concave shape of the curve describing the variation of compliance; in the first part of the curve corresponding to the first stage of jaw motion: approaching the targeted object, the gradient of compliance increase is small, while at the end of stroke compliance increases abruptly. When clamping between the jaws is achieved, the compliance of the gripper system needs to reach its highest values, thus allowing manipulation without the risk of deforming/ destroying the seized object.

An adjustable compliant motor can adapt its operational behaviour between two limits, ranging from rigid - for high accuracy positioning - to compliant, when the primary desired result is not precision, but safety of the motion [15]. A compliant actuator, like the pneumatic muscle, allows deviations from the position of equilibrium of a magnitude depending on the external forces the actuated element is subjected to [4].

Structures that can go from soft to rigid and viceversa (adjustable compliant ones) are used in this paper in order to design a novel gripper system for assembly operations. The gripper system includes a pneumatic muscle as a motor and a power transmission mechanism consisting of gears and racks.

\section{THE CONSTRUCTION OF THE GRIPPER SYSTEM}

While pneumatic muscles, as adjustable compliant actuators, are used mostly in applications where the safety of man-robot interactions needs to be ensured with priority, their deployment in other industrial applications is not excluded. An example of such application is assembling, such as introducing a peg into a hole (Fig. 2).

If the two components are not coaxial, the presence of a compliant assembling system is required such as to allow the compensation of the deviations. In order to facilitate the assembling, guiding chamfers are to be machined either at the upper part of the 
hole or the extremity of the peg or at the end of both components.
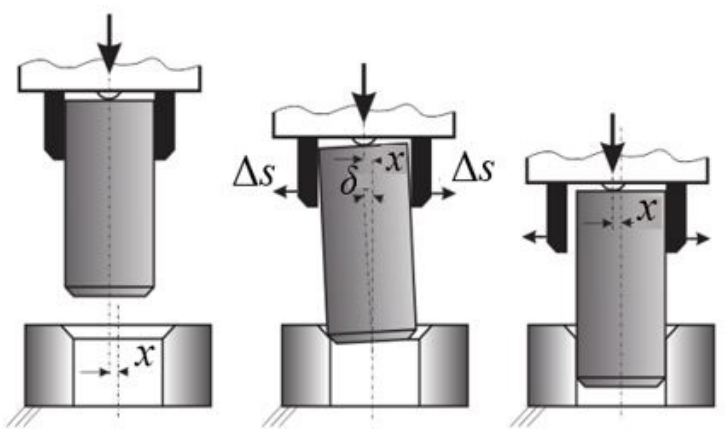

Fig. 2. Schematic of self-adaptive assembling

The figure shows that initially the peg and the hole are not coaxial. As soon as the front end of the peg makes contact with the hole's chamfer, the jaws of the gripper system are capable of displacement such as to compensate the misalignment. This, however, is possible only in case of a compliant actuation system of the jaws.

The gripper proposed in this paper is an asymmetrical two-jaw system, with a gear mechanism for power transmission. The asymmetry of the gripper system is given by the asymmetrical positioning of the pneumatic muscle in relation to the symmetry axis of the seized object, which allows fitting the proportional pressure regulator into the gripper body. In contrast, the asymmetry of the gripper is also given by the nonidentical structures of the two component linkages, the former including one intermediary gear and the latter two intermediary gears.

The gripper system was dimensioned based on the following input data: mass of the object $\mathrm{m}=0.7 \mathrm{~kg}$; acceleration of the motion of the system consisting of the gripper and the object: $a=5 \mathrm{~m} / \mathrm{s}^{2}$; gravitational acceleration: $g=9.81 \mathrm{~m} / \mathrm{s}^{2}$; emergency stop deceleration: $a_{S}=10 \mathrm{~m} / \mathrm{s}^{2}$; friction coefficient: $\mu=0.2$; safety coefficient: $S=2.5$. Taking into account the imposed requirements related to functioning and overall dimensions, the model proposed in this paper uses the smallest pneumatic muscle, (MAS-10-45NAA-MC-O-ER-EG), with an interior diameter of 10 $\mathrm{mm}$ and an active length of $45 \mathrm{~mm}$.

The maximum forces that have to be developed by this pneumatic muscle are generated when the accelerations are maximum, namely in sudden braking. In such case, the necessary gripping force of one jaw is computed by Eq. (1):

$$
\begin{aligned}
F_{\text {jaw }} & =\frac{m \cdot\left(g+a_{S}\right) \cdot S}{\mu \cdot n}= \\
& =\frac{0.7 \cdot(9.81+10) \cdot 2.5}{0.2 \cdot 2}=86.67 \mathrm{~N} .
\end{aligned}
$$

The construction of the proposed gripper system has two jaws; therefore, the selected pneumatic muscles have to be able to develop a force of minimum $2 \times 86.67 \mathrm{~N}=173.34 \mathrm{~N}$. Fig. 3 presents the graph that describes the evolution of the force developed by the selected pneumatic muscle versus charging pressure and stroke. The hachured rectangle highlights the optimum deployment area of the pneumatic muscle, so that for a maximum stroke of the jaws of $4 \mathrm{~mm}$, the developed force exceeds $173.34 \mathrm{~N}$.

Fig. 4 shows a view of the gripper system and Fig. 5 illustrates its pneumatic control diagram. The pneumatic control diagram of the pneumatic muscle includes a proportional pressure regulator (MPPES-3-1/4-6-010), controlled by a reference module MPZ-1-24DC-SGH-6-SW.

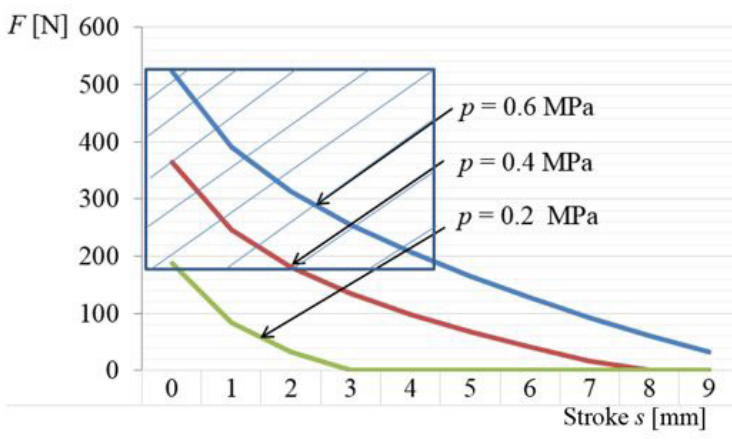

Fig. 3. Variation of the developed force versus the charging pressure and stroke

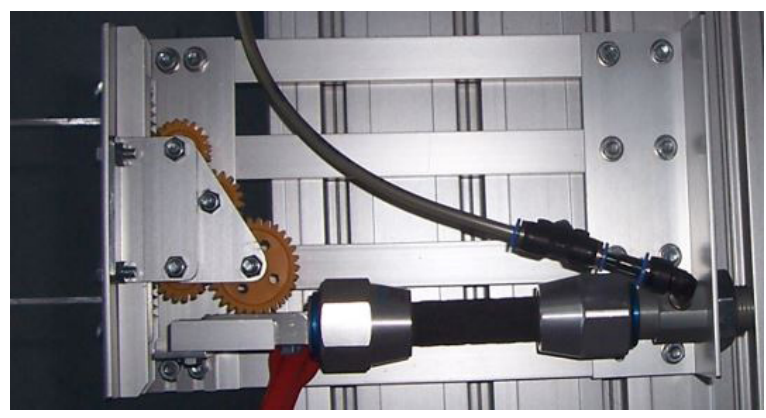

Fig. 4. Construction of the gripper system

Using rotational potentiometers, the reference module can generate up to six different values of the reference voltage, which are transmitted in form of signals to the proportional pressure regulator. If none of these reference values is used, the signal transmitted 
to the pressure regulator is a voltage adjustable via an external potentiometer. The continuous adjustment of the air pressure allows for modifying the compliance of the entire system.

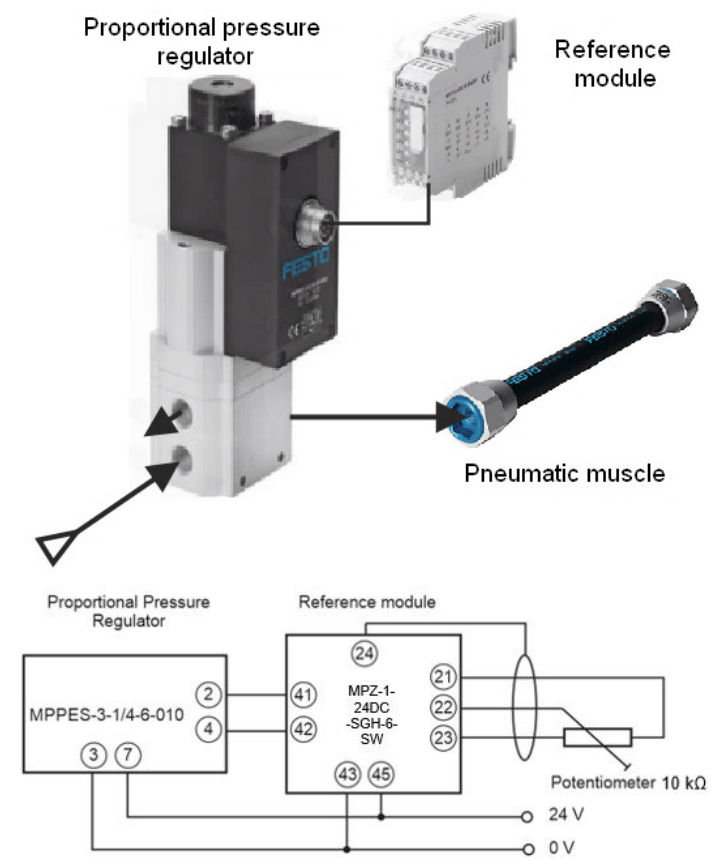

Fig. 5. Control of the gripper system by means of a proportional pressure regulator

The structural and block diagrams of the gripper system are presented in Fig. 6.
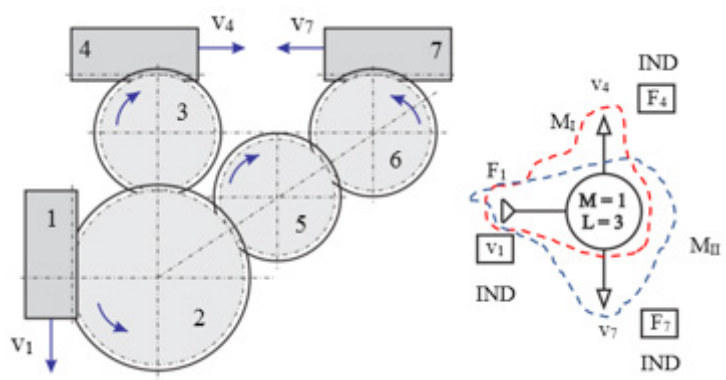

Fig. 6. Structural and block diagrams of the gripper system

The mechanism consists of two parallelconnected linkages $\left(\mathrm{M}_{\mathrm{I}}\right.$ and $\left.\mathrm{M}_{\mathrm{II}}\right)$ that branch out at gear 2. The motion of the entire system is generated by the pneumatic muscle and is characterized by a velocity $\left(\mathrm{v}_{1}\right)$ and a force $\left(\mathrm{F}_{1}\right)$ of the same orientation. The system outputs are its exterior links to the gripper jaws, and are characterized each by a velocity ( $\mathrm{v}_{4}$ and $\mathrm{v}_{7}$ ) and a force $\left(\mathrm{F}_{4}\right.$ and $\left.\mathrm{F}_{7}\right)$, of opposite directions.

The characteristics of the gears and racks used in the construction of the gripper system are: racks 4 and
$7:$ module $=1 \mathrm{~mm}$; number of teeth of the gears: $\mathrm{z}_{2}=$ $30 ; \mathrm{z}_{3}=\mathrm{z}_{5}=\mathrm{z}_{6}=20$.

If the friction occurring in the mechanism is not neglected, the efficiency of each gear has to be included in the computation of the transmission function of the forces. The following efficiencies were considered for the analysed gripper system: $\eta_{12}=\eta_{34}$ $=\eta_{67}=0.97 ; \eta_{23}=\eta_{25}=\eta_{56}=0.95$.

According to how the mechanisms are linked, the analysed gripper system is a mixed type aggregate, in which the two series-type mechanisms $\mathrm{M}_{\mathrm{I}}$ and $\mathrm{M}_{\mathrm{II}}$ are parallel connected starting from the power node at gear 2. As the number of mechanisms composing the two branches is not equal, the studied aggregate is of the non-homogeneous mixed type. An energetically equivalent homogenous diagram can with associated to this aggregate, presented in Fig. 7. The power at the system input is denoted by $\mathrm{N}$, while $\mathrm{N}_{\mathrm{u} 1,2}$ are the useful powers obtained at the outputs of the branches of the aggregate.

The efficiency of the gripper system was determined by matrix calculus. An array containing the coefficients $\beta$ associated to the distribution onto branches of the output powers, and the partial efficiencies ordered by the connection of the component linkages was attached to the analysed mechanism. This array is called an associated matrix and takes the form of Eq. (2) for the studied aggregate:

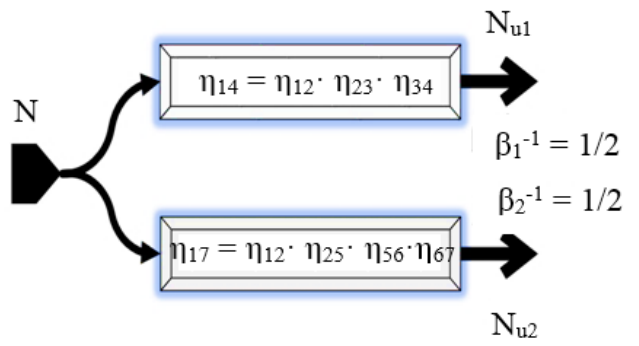

Fig. 7. Homogenous diagram associated to the gripper system

$$
\left[\mathrm{M}_{\beta}\right]=\left[\begin{array}{ccccc}
\beta_{1}^{-1} & \eta_{12}^{-1} & \eta_{23}^{-1} & \eta_{34}^{-1} & 1 \\
\beta_{2}^{-1} & \eta_{12}^{-1} & \eta_{25}^{-1} & \eta_{56}^{-1} & \eta_{67}^{-1}
\end{array}\right]
$$

The number of rows of the matrix corresponds to the branches of the aggregate, while the number of columns is that of the maximum number of gears included by the branches +1 . Thus, a $2 \times 5$ matrix resulted. Based on this matrix, the efficiency of the aggregate can be determined by Eq. (3):

$$
\left[\eta^{-1}\right]=\sum_{C}\left(\prod_{l}\left[\mathrm{M}_{\beta}\right]\right)
$$


where the following two operators have been introduced:

- Product by row operator $\prod\left[\mathrm{M}_{\beta}\right]$ :

$$
\prod_{1}\left[\mathrm{M}_{\beta}\right]=\left[\begin{array}{c}
\beta_{1}^{-1} \cdot \eta_{12}^{-1} \cdot \eta_{23}^{-1} \cdot \eta_{34}^{-1} \cdot 1 \\
\beta_{2}^{-1} \cdot \eta_{12}^{-1} \cdot \eta_{25}^{-1} \cdot \eta_{56}^{-1} \cdot \eta_{67}^{-1}
\end{array}\right] .
$$

- Sum by column operator $\sum_{C}$ :

$$
\sum_{C}\left[\begin{array}{l}
\beta_{1}^{-1} \cdot \eta_{12}^{-1} \cdot \eta_{23}^{-1} \cdot \eta_{34}^{-1} \cdot 1+ \\
\beta_{2}^{-1} \cdot \eta_{12}^{-1} \cdot \eta_{25}^{-1} \cdot \eta_{56}^{-1} \cdot \eta_{67}^{-1}
\end{array}\right] .
$$

By entering the values of the efficiencies of each elementary mechanism, the efficiency of the system will be computed in the following steps:

$$
\begin{aligned}
& {\left[\mathrm{M}_{\beta}\right]=\left[\begin{array}{ccccc}
0.5 & 0.97^{-1} & 0.95^{-1} & 0.97^{-1} & 1 \\
0.5 & 0.97^{-1} & 0.95^{-1} & 0.95^{-1} & 0.97^{-1}
\end{array}\right] \text {, }} \\
& \prod_{l}=\left[\begin{array}{c}
0.5 \cdot 0.97^{-1} \cdot 0.95^{-1} \cdot 0.97^{-1} \cdot 1 \\
0.5 \cdot 0.97^{-1} \cdot 0.95^{-1} \cdot 0.95^{-1} \cdot 0.97^{-1}
\end{array}\right]=\left[\begin{array}{c}
0.5593 \\
0.5888
\end{array}\right] \text {, } \\
& \sum_{C}\left(\prod_{l}\left[\mathrm{M}_{\beta}\right]\right)=[0.5593+0.5888]=1.1481, \\
& \rightarrow \eta=1.1481^{-1}=0.8709 .
\end{aligned}
$$

Knowing the value of the theoretical global efficiency, the equilibrium of powers can be written in the form of Eq. (6):

$$
F_{1} \cdot v_{1} \cdot \eta+F_{4} \cdot v_{4}+F_{7} \cdot v_{7}=0
$$

where from follows the transmission function of the forces:

$$
\begin{aligned}
F_{1} & =\frac{1}{\eta}\left(-F_{4} \cdot \frac{v_{4}}{v_{1}}-F_{7} \cdot \frac{v_{7}}{v_{1}}\right)= \\
& =\frac{1}{\eta}\left(-F_{4} \cdot \frac{1}{i_{14}}-F_{7} \cdot \frac{1}{i_{17}}\right)=\frac{1}{\eta}\left(F_{4}-F_{7}\right), \\
& \rightarrow F_{1}=\frac{F_{4}-F_{7}}{0.8709} .
\end{aligned}
$$

\section{EXPERIMENTAL RESULTS}

The experimental research was focused on the study of the entire assembly, such as to determine the force generated at the jaws and the compliance of the system.

The maximum force that can be developed by one jaw was measured by the attached force sensor (Fig. 8).

The magnitude of this force depends, as was the case of the jaw's stroke, not only on the pressure but also on the direction of pressurization (increasing/ inflation or decreasing/deflation). Fig. 9 shows the evolution of the force developed by a jaw versus pressure, when the pneumatic muscle is charged (inflated) and discharged (deflated), respectively.

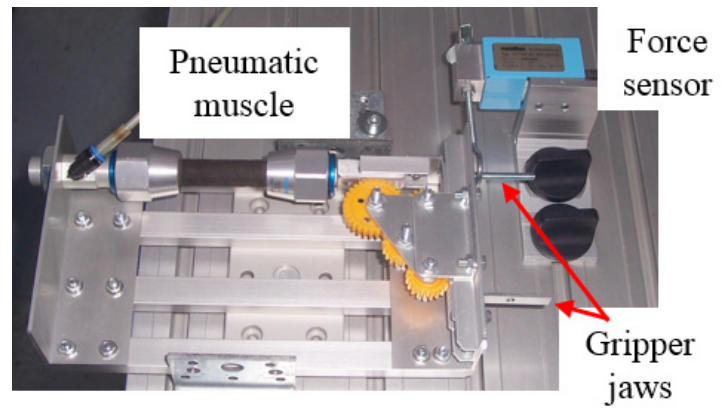

Fig. 8. Force measurement developed by one jaw

The graphs reveal a strong hysteresis type behaviour of the developed force. It can be observed that during the charging of the muscle the force starts increasing only beyond 1.318 bar pressure, and reaches its maximum of $83.33 \mathrm{~N}$ at 6 bar.
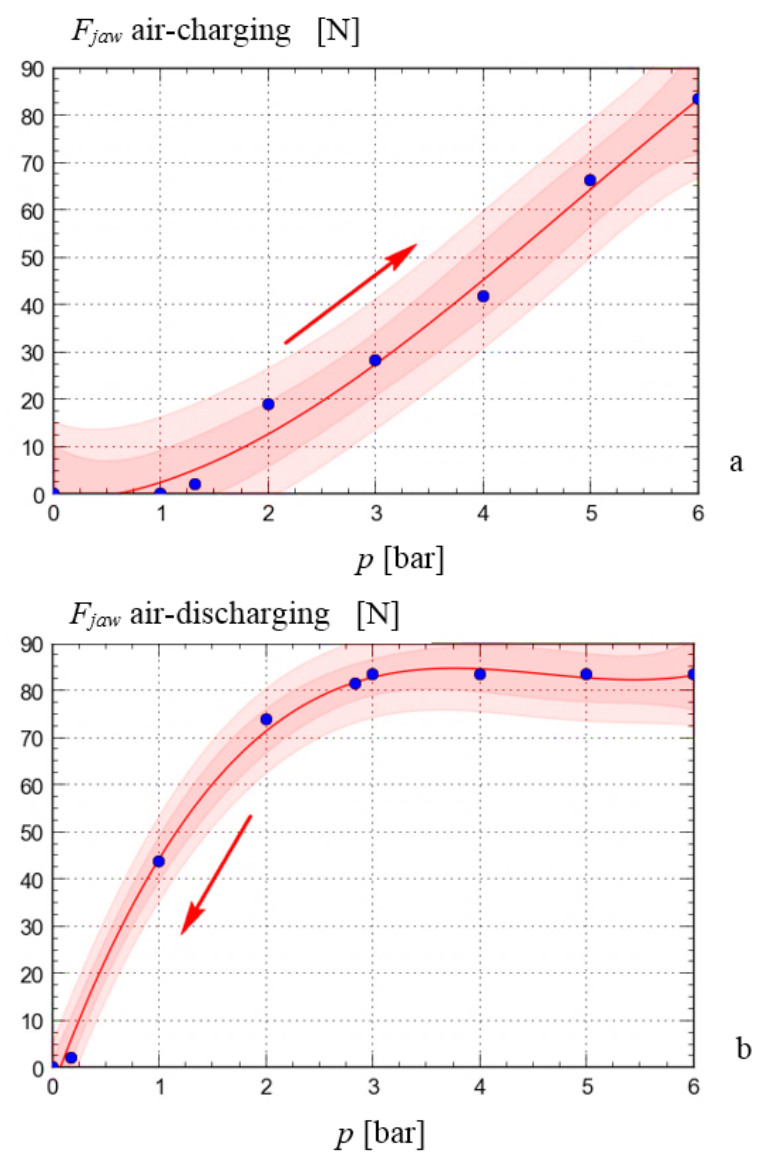

Fig. 9. The force developed by one jaw for pressure: a) charging, and b) discharging 
During the discharging of the muscle, the force at first holds its maximum value, and starts decreasing only beneath 2.9 bar pressure, reaching $0 \mathrm{~N}$ at 0.15 bar.

The occurrence of hysteresis during operation is a major disadvantage of pneumatic muscles. In essence, hysteresis is a loss of energy, and a source of error difficult to control in a system that requires high positioning accuracy.

The hysteresis of pneumatic muscles is caused by the deformation of the elastic tube, as well as by the internal friction between each aramid fibre and its elastic envelope. These phenomena generate a so-called threshold pressure that translates into a stay (stagnation) of the pneumatic muscle's axial deformation, even if the feed pressure varies.

The hysteresis of pneumatic muscles increases the non-linearity of the systems in which they are included and adds to the complexity of the control systems.

In the case of the analysed gripper system of interest is the curve corresponding to the inflation of the pneumatic muscle, i.e. when the standard phases of the gripping process described in Fig. 1 are carried out. The threshold pressure (at 1.318 bar) narrows the useful area of the working pressures: only after this threshold value is exceeded will the gripping force move away from zero thus allowing the seizing of the targeted object.

The second curve corresponding to the deflation of the pneumatic muscle has an insignificant influence on the gripping process. It can be noticed that while the pressure decreases from 6 bar to 2.9 bar the force exerted by the jaw does not change (does not decrease); it is only beyond this value that the object is released. Thus, there is a certain delay (lag) in the system response (releasing of the object) to the command (decrease of the pressure).

Fig. 10 shows the variation of the force developed by one jaw versus its stroke. It can be noticed that at the beginning of the motion the force is maximum $(83.33 \mathrm{~N})$, and that it decreases as the jaw nears the end of its stroke.

Eq. (8) describes the regression function corresponding to the curve displayed above with a correlation coefficient of 0.999997 :

$$
F_{\text {jaw }}=83.36-19.146 \cdot s+0.7088 \cdot s^{2},
$$

where $s$ denotes the stroke of one jaw.

The obtained experimental results also allow the determination of the global efficiency of the analysed gripper system, to be matched against the theoretically

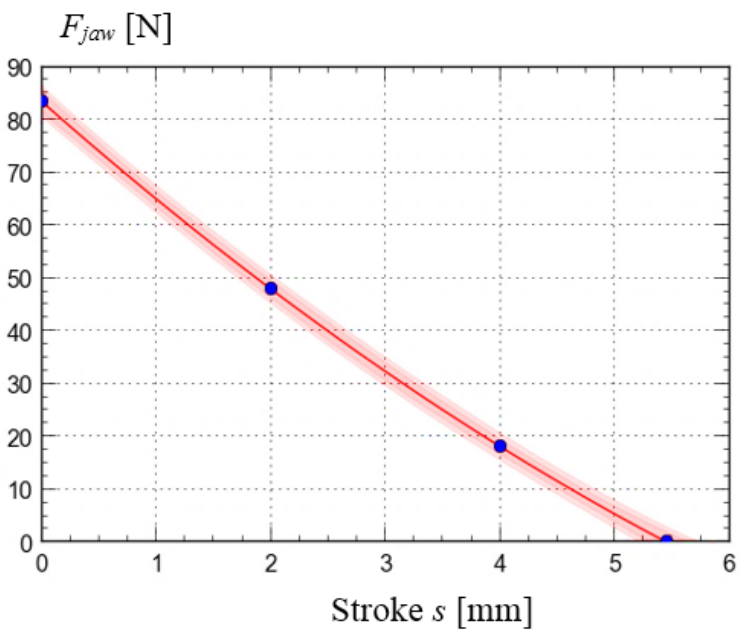

Fig. 10. Variation of the force developed by one jaw versus its stroke

computed value $\left(\eta_{\text {theoretical }}=0.8709\right)$. Thus, depending on the measured forces, the efficiency of the entire gripper system will be:

$$
\eta_{\text {real }}=\frac{2 \cdot F_{\text {jaw }}}{F_{i}}=\frac{2 \cdot 83.33}{187.4}=0.8893,
$$

where $F_{i}=187.4 \mathrm{~N}$ is the value of the maximum force developed by the selected pneumatic muscle (measured value). This value of the efficiency is very close to the theoretically obtained one.

The stiffness $k$ of the analysed system is computed by Eq. (9), and compliance $C$ is determined as the inverse of stiffness.

$$
\begin{gathered}
k=-\frac{d F}{d s}=19.146-1.4176 \cdot s, \\
C=k^{-1}=\left(-\frac{d F}{d s}\right)^{-1}=\frac{1}{19.146-1.4176 \cdot s} .
\end{gathered}
$$

Fig. 11 presents the graphs of the stiffness and the compliance of the analysed gripper system. The concave shape of the curve describing the variation of the compliance ensures an enhanced growth of the compliance towards the end of the jaws' stroke, exactly at the beginning of contact with the gripped object and in accordance with the requirements specified in Fig. 1. The compliance of the gripper system grows as the stroke of the jaw advances, thus ensuring safe and soft gripping, without the risk of deforming the seized object.

Fig. 12 illustrates the dependence of the gripper system stiffness and compliance on the stroke of one jaw and on the air pressure. According to these 


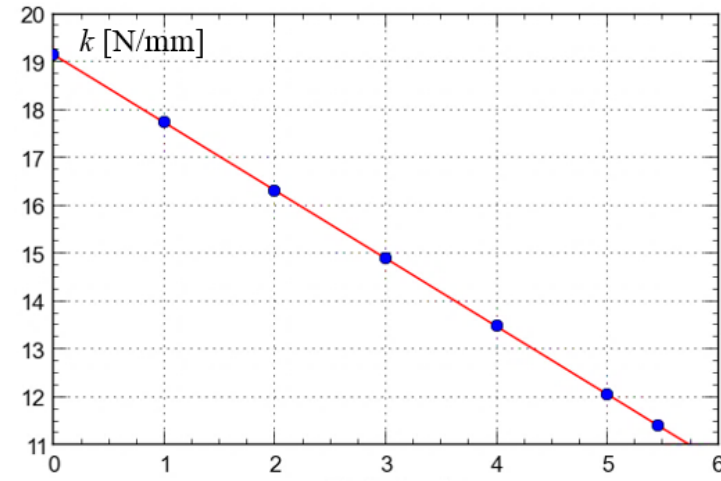

a)

Stroke $s[\mathrm{~mm}]$

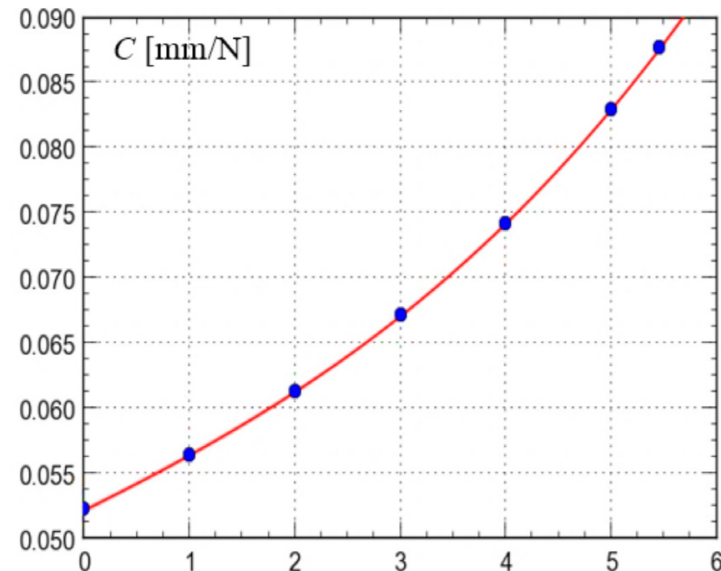

b)

Stroke $s[\mathrm{~mm}]$

Fig. 11. Variation of the gripper system: a) stiffness and b) compliance

figures, the studied gripper system is characterized by decreasing stiffness and increasing compliance as the jaw's stroke advances and pressure grows. Such an evolution of stiffness and compliance results in a longer response time of the system to load variations, and evidently in smaller accuracy, which may represent an inconvenience. In contrast, in applications where system rigidity is less important, the main requirement being that of safe handling of the gripped object, the possibility of adjusting compliance so that it increases with pressure most certainly represents and advantage.

The functions describing the stiffness and the compliance of the studied gripper are:

$$
\begin{aligned}
k & =19.146-0.000346 \cdot p-1.417 \cdot s, \\
C & =0.0522+0.00011 \cdot p+0.00232 \cdot s+ \\
& +0.000132 \cdot p^{2}+0.000581 \cdot s^{2} .
\end{aligned}
$$

The concrete data collected by the conducted measurements further allow defining intervals of
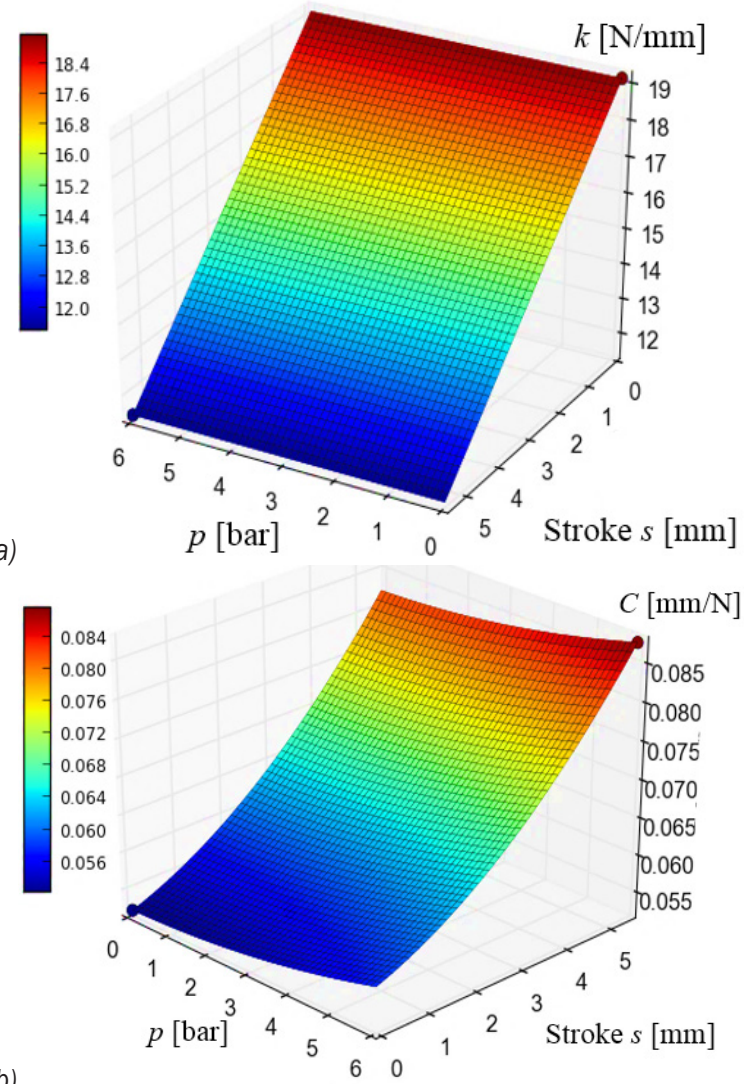

Fig. 12. Variation of the gripper system a) stiffness and b) compliance versus pressure and stroke

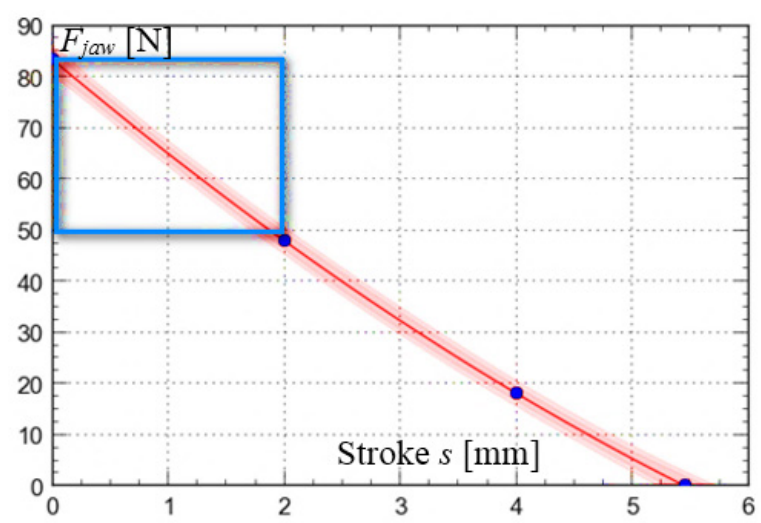

Fig. 13. Identification of the area of interest in the deployment of the gripper system

forces and strokes of the jaw, recommended for the deployment of the gripper system.

Thus, Fig. 13 presents the curve describing the variation of the force developed by one jaw versus its stroke. An area of interest is identified in this diagram where forces ranging from 50 to $83.33 \mathrm{~N}$ are obtained, at jaw strokes up to $2 \mathrm{~mm}$. 
For this functional area, the masses of the objects that can be seized range from $0.4 \mathrm{~kg}$ (for a $2-\mathrm{mm}$ stroke) to $0.7 \mathrm{~kg}$ (for a $0-\mathrm{mm}$ stroke).

\section{CASE STUDY}

Fig. 14 presents the case of assembling a nonchamfered peg into a chamfered hole, the two components being axially misaligned.

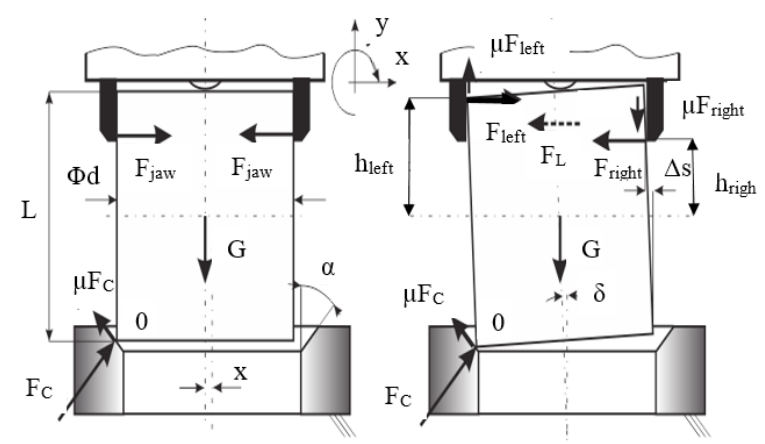

Fig. 14. Assembling of radially misaligned components

The peg is moved to the assembly zone by the jaws of the gripper system, until its edge touches the surface of the hole's chamfer. At this point, a contact force $F_{C}$ is generated that tends to rotate the peg so as to allow assembly. This is not achievable unless the peg's gripper system is a compliant one, as is the one actuated by pneumatic muscles.

The tilt of the peg of angle $\delta$ causes additional forces to appear at the jaws, denoted by $F_{\text {left }}$ and $F_{\text {right }}$. It is their cumulative effect $\left(F_{L}=F_{\text {left }}-F_{\text {right }}\right)$ that allows a small displacement $(\Delta s)$ of the two jaws, and consequently the turning of the peg by angle $\delta$. In order to determine the additional displacement $\Delta s$ of the two jaws, the following numerical case will be considered: $d=47 \mathrm{~mm} ; L=50 \mathrm{~mm} ; \alpha=45^{\circ} ; m=0.7 \mathrm{~kg}$; $g=9.81 \mathrm{~m} / \mathrm{s}^{2} ; \mu=0.2 ; h_{\text {left }}=25 \mathrm{~mm} ; h_{\text {right }}=15 \mathrm{~mm}$.

Furthermore, the equations of equilibrium of the forces and torques developed in the system are written:

$$
\begin{gathered}
\sum F_{x}=0 \\
F_{C} \cdot \cos \alpha-\mu \cdot F_{C} \cdot \sin \alpha+F_{\text {left }}-F_{\text {right }}=0, \\
\sum F_{y}=0 \\
F_{C} \cdot \sin \alpha+\mu \cdot F_{C} \cdot \cos \alpha+\mu \cdot F_{\text {left }}-\mu \cdot F_{\text {right }}-G=0,( \\
\sum M_{0}=0 \\
F_{\text {left }}\left(h_{\text {left }}+\frac{L}{2}\right)+G \frac{d}{2}-F_{\text {right }}\left(h_{\text {right }}+\frac{L}{2}\right)+ \\
+\mu \cdot F_{\text {right }} \cdot d=0 .
\end{gathered}
$$

For the above input data, the following results were obtained upon solving of the system of equations: $F_{\text {right }}=5.3 \mathrm{~N} ; F_{\text {left }}=0.02 \mathrm{~N}$; $F_{L}=F_{\text {left }}-F_{\text {right }}=-5.28 \mathrm{~N}$.

The resulting lateral force $F_{L}$ is the one that allows a small displacement (retraction) of the system's jaws. Thus, the clamping force of the handled object is slightly diminished, and the peg, due to its own weight and the pushing force developed by the gripper system is introduced into the hole.

Starting from Eq. (10) the additional displacement of the jaws can be determined. This is computed by Eq. (16):

$$
\Delta s=\frac{F_{L}}{19.146-1.4176 \cdot s} .
$$

Fig. 15 shows the corresponding graph.

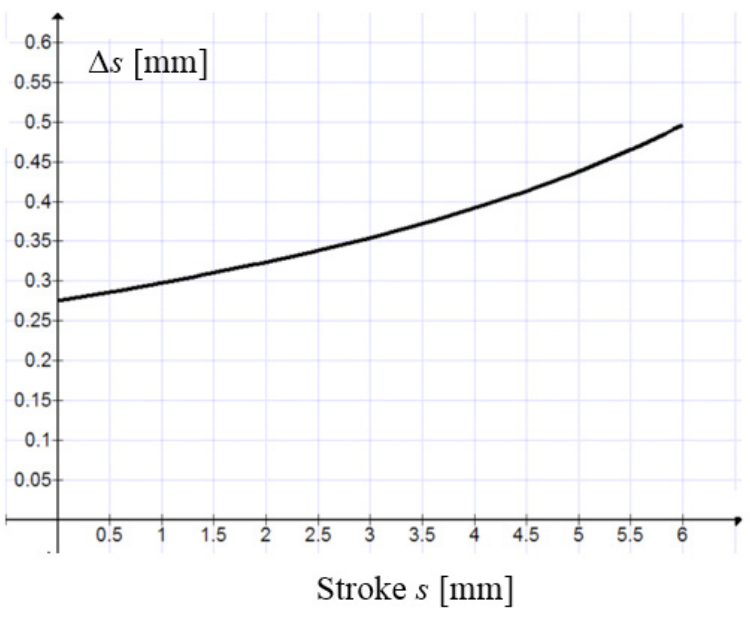

Fig. 15. Magnitude of one jaw's radial displacement versus its stroke

The conclusion following from the above graph is, that as the stroke carried out by the jaw becomes greater and implicitly the system's compliance grows, the additional displacements can be correspondingly greater. This allows working with a lesser positioning accuracy of the robot and with increased misalignments of the mating components, without compromising the assembling process.

\section{CONCLUSIONS}

The obtained experimental results reveal that the analysed gripper system is characterized by a behaviour strongly affected by hysteresis. The hysteresis of the pneumatic muscle is caused by the friction occurring between the enveloping tissue and the elastic tube, by the inner friction between 
the threads of the enveloping tissue, as well as by the non-elastic deformation of the inner tube. This disadvantage carried by the behaviour of the entire system renders pneumatic muscle based actuations feasible only when the required precision of the gripping is not very high.

The measurements yielded a non-linear dependency of the developed force on the displacement for this gripper system. This means that the system has adjustable compliance, which renders it eligible for assembling applications. In this case, when two parts to be assembled are not perfectly aligned, a compliant system, like the one presented and discussed in this paper, offers the benefit of behaviour adaptable to the given situation, allowing assembling without destroying the two components.

As a conclusion to the conducted research, it can be asserted that due to their specific behaviour pneumatic muscles represent a viable constructive alternative for modern gripper systems.

\section{REFERENCES}

[1] Fantoni, G., Gabelloni, D., Tilli, J. (2012). How to Design New Grippers by Analogy, Report. University of Pisa, Pisa.

[2] Negrea, D., Deaconescu, T., Deaconescu, A. (2014). Symmetrical pneumatic muscle actuated gripper system with two mobile jaws. Applied Mechanics and Materials, vol. 541542, p. 852-856, D0l:10.4028/www.scientific.net/AMM.541542.852.

[3] Stăncescu, C. (2009). Theoretical and Experimental Study of Grippers Systems with Jaws used in Industrial Robots. PhD thesis, Transilvania University of Braşov, Braşov.

[4] Van Ham, R., Sugar, T.G., Vanderborght, B., Hollander, K.W., Lefeber, D. (2009). Compliant Actuator Designs. IEEE Robotics \& Automation Magazine, vol. 16, no. 3, p. 81-94, D0l:10.1109/ MRA.2009.933629.
[5] Petkovic, D., Isac, M., Pavlovic, N.D., Zentner, L. (2012). Passively adaptive compliant gripper. Applied Mechanics and Materials, vol. 162, p. 316-325, D0l:10.4028/www.scientific. net/AMM.162.316.

[6] Deimel, R., Brock, O. (2013). A compliant hand based on a novel pneumatic actuator. IEEE International Conference on Robotics and Automation, D0l:10.1109/ICRA.2013.6630851.

[7] Christopoulos, V.N., Schrater, P.R. (2009). Grasping objects with environmentally induced position uncertainty. PLOS Computational Biology, vol. 5, no. 10, p. 538, D0l:10.1371/ journal.pcbi.1000538.

[8] Ciocarlie, M., Hicks, F.M., Stanford, S. (2013). Kinetic and Dimensional Optimization for a Tendondriven Gripper. IEEE International Conference on Robotics and Automation.

[9] Dollar, A.M., Howe, R.D. (2010). the highly adaptive SDM hand: design and performance evaluation. The International Journal of Robotics Research, vol. 29, no. 5, p. 585-597, DOl:10.1177/0278364909360852.

[10] Monkman, G.J., Hesse S., Steinmann, R., Schunk, H. (2007). Robot Grippers. Wiley-VCH Verlag, Weinheim.

[11] Deaconescu, A., Deaconescu, T. (2011). Bio-inspired pneumatic muscle actuated robotic system. Intelligent Automation and Systems Engineering, Springer New York, p. 27-40, DOI:10.1007/978-1-4614-0373-9_3.

[12] Festo. Power Gripper, from http://www.festo.com/net/ SupportPortal/Files/156738/Brosch_FC_PowerGripper_EN_ 10_L.pdf, accessed on 2015-12-07.

[13] Negrea, D., Deaconescu, A., Deaconescu, T. (2014). Constructive and functional modelling of a pneumatic muscle actuated symmetric gripper system with two mobile jaws. Applied Mechanics and Materials, vol. 657, p. 574-578, D0I:10.4028/www.scientific.net/AMM.657.574.

[14] Deaconescu, T., Deaconescu, A. (2015). Structural, kinematic and static modelling of a pneumatic muscle actuated gripper system. Applied Mechanics and Materials, vol. 811, p. 318322, D0I:10.4028/www.scientific.net/AMM.811.318.

[15] Bicchi, A., Tonietti, G. (2004). Fast and soft arm tactics: dealing with the safety-performance trade-off in robot arms design and control. IEEE Robotics and Automation Magazine, vol. 11, no. 2, p. 22-33, D0I:10.1109/MRA.2004.1310939. 